\title{
Focality and Asymmetry in Multi-battle Contests
}

\author{
Subhasish M. Chowdhury ${ }^{\mathrm{a}}$, Dan Kovenock ${ }^{\mathrm{b}}$, \\ David Rojo Arjona ${ }^{\mathrm{c}}$, and Nathaniel T. Wilcox ${ }^{\mathrm{b}}$ \\ ${ }^{a}$ School of Economics, Centre for Behavioural and Experimental Social Science, and Centre for Competition Policy, University of East Anglia, Norwich \\ NR4 7TJ, UK. \\ ${ }^{\mathrm{b}}$ Economic Science Institute, Chapman University, One University Drive, Orange, CA 92866, USA. \\ ${ }^{c}$ Department of Economics, University of Leicester, University Road, Leicester, LE1 7RH, UK.
}

August 16, 2016

\begin{abstract}
This article examines behavior in two-person constant-sum Colonel Blotto games in which each player maximizes the expected total value of the battlefields won. A lottery contest success function is employed in each battlefield. Recent experimental research on such games provides only partial support for Nash equilibrium behavior. We hypothesize that the salience of battlefields affects strategic behavior (the salient target hypothesis). We present a controlled test of this hypothesis - against Nash predictions - when the sources of salience come from certain asymmetries in either battlefield values or labels (as in Schelling (1960)). In both cases, subjects over-allocate the resource to the salient battlefields relative to the Nash prediction. However, the effect is stronger with salient values. In the absence of salience, we replicate previous results in the literature supporting the Nash prediction.
\end{abstract}

JEL Classifications: C72, C91; D72; D74

Keywords: Conflict; Experiment; Colonel Blotto, Focal point, Asymmetry

\footnotetext{
${ }^{*}$ Corresponding author: Dan Kovenock (kovenock@chapman.edu).

We thank Kai Konrad, Jason Sachat, Roman Sheremeta, Robert Sugden and Daniel Zizzo for helpful comments. We have also benefited from the comments of participants in seminars at Calcutta, Chapman, Durham/Newcastle, East Anglia and Middlesex, in workshops on Strategic Aspects of Terrorism, Security and Espionage at Stony Brook University, Current Frontiers in Theory of Contests at the Max Planck Institute for Tax Law and Public Finance, Advances in the Economics of Conflict at the Stockholm School of Economics, and at the $16^{\text {th }}$ SAET Conference in Rio de Janeiro. David Rojo Arjona acknowledges generous funding from the Economic Science Institute at Chapman University.
} 


\section{Introduction}

This article experimentally examines the influence of "focality" or "salience"1 on individual behavior in a class of multi-battle contests known as the Colonel Blotto game with a lottery contest success function. In this game, two players simultaneously allocate their respective endowments, or "budgets", of a one dimensional resource (such as effort, money, time, or troops) across a set of "battlefields". Each player's allocation to a given battlefield determines the likelihood that the player wins the battlefield, with the probability of winning given by the player's own expenditure divided by the sum of the two players' expenditures (hence, the term "lottery"). ${ }^{2}$

Colonel Blotto games and other closely related multi-battle contests are widely applied in the formal modeling of strategic behavior in the context of military operations, network and infrastructure security, and anti-terrorism defense. ${ }^{3}$ In these environments, the salience of specific battlefields or targets often influences the strategic allocation of resources in the conflict. We call this observation the salient target hypothesis (STH). For instance, during the US military invasion of Iraq in 2003, the capture of high profile targets such as Saddam Hussein and the killing of his sons, Uday and Qusay were viewed as having significant symbolic value. Similarly, the killing of Abu Musab al-Zarqawi was viewed as having higher symbolic than tactical benefit. In the context of terrorism, Nemeth (2010, p.30) argues that the 2000 suicide attack on the USS Cole in the Yemeni port of Aden involved a well-defended target that had high symbolic value despite the relatively few casualties involved. More well-known attacks on salient targets include the Black September terrorist attack on the Israeli Olympic team at the 1972 Munich Olympics and the September 11, 2001 al-Qaeda terrorist attacks on the World Trade Center and the Pentagon. In the context of information network security, young hackers often gain fame by attacking salient targets. In 1999, Jonathan James became notorious for his hack of NASA and The US Department of Defense. In 2000, Michael Calce (a.k.a. MafiaBoy) became famous for his distributed denial of service attacks on Amazon, CNN, eBay and Yahoo!.

In each of these cases it is clear that the salience of a target by itself induces a higher value to successful attack than less salient targets. For instance, in the case of military campaigns a high profile victory may have positive implications for morale at home and induce an enemy population to become discouraged. High profile terrorist attacks may induce fear in the intended target population, place pressure on the

\footnotetext{
${ }^{1}$ The terms focality, salience, prominence are used almost interchangeably in the literature. There is a tendency to refer to focal points or focality when referring to behavior and salience or prominence when describing an aspect or element of the game that generates that behavior. In this paper we will conform to this usage.

${ }^{2}$ Randomization is independent across battlefields and in the event of zero aggregate expenditure to a battlefield, the probability that each player wins is $\frac{1}{2}$.

${ }^{3}$ See, for instance, Shubik and Weber (1981), Powell (2007a,b, 2009), Powers and Shen (2009), Kovenock et al. (2010), Chia and Chuang (2011), Arce et al. (2011), Fuchs and Khargonekar (2012), Nikoofal and Zhuang (2012), Arce et al. (2012), Bachrach et al. (2013), Gupta et al. (2014a,b), Hausken (2014), Goyal and Vigier (2014). Other applications include the optimal allocation of advertising budgets across markets or marketing budgets across channels (Friedman, 1958), the allocation of political campaign funds across different primaries or state races (Snyder, 1989; Laslier, 2002; Klumpp and Polborn, 2006; Kovenock and Roberson, 2009a), redistributive politics (Myerson, 1993; Lizzeri and Persico, 2001; Roberson, 2008; Kovenock and Roberson, 2008, 2009b; Crutzen and Sahuguet, 2009), and the allocation R\&D budgets across several potential classes of innovations or projects(Clark and Konrad, 2008). See Kovenock and Roberson (2012b) for a review.
} 
target governments, and aid in both fundraising and recruiting among potential supporters. Despite this perceived positive association between the salience of a target and higher value, it is not clear whether the levels of resources allocated to attack such targets are optimal given the induced value of the target, or whether the salience of the target itself has an influence independent of its induced value. In fact, it appears very difficult to even address this question with case studies or other data from the field, since real-world combatants actual target values are unobservable. The purpose of this article is to investigate the salient target hypothesis by carrying out a laboratory experiment that separates out the positive effects of target value and salience in the determination of resource allocation in multi-battle contests. To our knowledge, this article is the first to examine the joint role that value and salience play in influencing strategic behavior in such games.

The theoretical origin of the Colonel Blotto game traces back to Borel (1921), who anticipated its future application, particularly to military strategy. ${ }^{4}$ McDonald and Tukey (1949) refer to the use of Colonel Blotto games in military research at Princeton during World War II and a substantial amount of work on the game was undertaken in the late 40s and 50s in the context of military operations research (Gross and Wagner, 1950; Gross, 1950; Blackett, 1953, 1954, 1958). Since that time, work on the problem has been sporadic (including Bellman, 1969; Shubik and Weber, 1981), until very recently, when a resurgence of interest has taken place. ${ }^{5}$

In addition to this extensive theoretical literature, there is a young and rapidly growing experimental literature on Blotto games. Several existing contributions employ an auction contest success function to determine the winner of each battlefield. Some of these reward subjects based on the total number or value of battlefields won (Avrahami and Kareev, 2009; Arad and Rubinstein, 2012; Cinar et al., 2012; Chowdhury et al., 2013). Others reward subjects who win either a majority of the battlefields or more than half of the total value of battlefields (Arad, 2012; Montero et al., 2016; Mago and Sheremeta, 2012). Experimental evidence on multi-battle contests employing a lottery CSF is still limited. Chowdhury et al. (2013) examine Colonel Blotto games with a lottery CSF in which subjects are rewarded based on the number of battlefields won. Irfanoglu et al. (2013) carry out an analysis of the case where the reward is based on attaining a majority of the battlefields. Duffy and Matros (2013) examine Colonel Blotto games with values that are heterogeneous across battlefields but symmetric across players and treat both a reward structure linear in the value of battlefields won and one that rewards winning a critical value of more than half of the total value of the battlefields. ${ }^{6}$

\footnotetext{
${ }^{4}$ The original problem posed by Borel was not solved until Borel and Ville (1938).

${ }^{5}$ See, for instance, Robson (2005), Roberson (2006), Clark and Konrad (2007), Kvasov (2007), Powell (2007a,b), Hart (2008), Roberson (2008), Golman and Page (2009), Adamo and Matros (2009), Kovenock et al. (2010), Kovenock and Roberson (2011), Hortala-Vallve and Llorente-Saguer (2012), Kovenock and Roberson (2012a), Roberson and Kvasov (2012), Rinott et al. (2012), Thomas (2012), Weinstein (2012), Rietzke and Roberson (2013), Hernández and Zanette (2013), Washburn (2013), Osorio (2013), McBride and Hewitt (2013), Sela and Erez (2013), Dziubiński (2013), Barelli et al. (2014), Gupta et al. (2014a,b), Goyal and Vigier (2014), Hart (2015), Schwartz et al. (2014), Macdonell and Mastronardi (2015), and Kovenock and Roberson (2015).

${ }^{6}$ Kovenock et al. (2010) also examine, under both the auction and lottery CSFs, the case in which players have asymmetric objectives; one player has a best-shot objective and the other a weakest-link objective.
} 
In this article we examine two-person, four-battlefield, constant-sum Colonel Blotto games in which each player earns the sum of the values of the battlefields that he wins, budgets are use-it-or-lose-it, and a lottery contest success function (Tullock, 1980) is employed in each battlefield. In this class of games, the unique Nash equilibrium is for the allocation to each battlefield to be proportional to the value of the battlefield and for the sum of the allocations to exactly exhaust the player's budget (Friedman, 1958). The recent experimental research on such games provides substantial support for Nash equilibrium behavior in games with values that are symmetric across both players and battlefields. Chowdhury et al. (2013) find support for the equilibrium prediction that each player should divide his budget equally across battlefields. ${ }^{7}$ Duffy and Matros (2013) claim qualitative but not quantitative support for the Nash equilibrium prediction in games in which battlefield values are symmetric across players, but not across battlefields. In light of this previous work, we believe that support for the equilibrium prediction in these studies, to the extent that it exists, arises from the absence of asymmetries in the battlefields that yields focal behavior. In line with the salient target hypothesis, deviations from the Nash prediction may arise in the presence of certain asymmetries in the primitives or visual representation of the game, such as battlefield values or labels.

We test this hypothesis by employing an experimental design in which subjects are randomly matched and face such asymmetries in each of these features, while keeping the other features symmetric. In the case of an asymmetric label, a single battlefield is colored differently from the others (black instead of white). In the case of an asymmetric value, one battlefield has a higher value than the others. We single out one battlefield (target) from the others to address a concern that arises in the econometric analysis of the game: because of the constraint that the sum of battlefield allocations must equal the subjects budget, a negative correlation of bids is induced across battlefields. In our experimental setting, all of the information about bidding behavior in the Blotto game collapses into statements about the bidding behavior in the target battlefield. This avoids the improper assumption of independence of allocations across battlefields.

In the completely symmetric case, we find strong support for Nash behavior. However, consistent with the salient target hypothesis, subjects deviate from Nash behavior in the presence of asymmetries in labels and battlefield values. With asymmetries, subjects over-allocate relative to the Nash equilibrium prediction in the saliently labeled and high value battlefields. Moreover, as might be expected given its payoff irrelevance, asymmetry in labels has a weaker effect than in values. With asymmetric values, the high value battlefield receives a significantly higher allocation than in the unique Nash equilibrium, even though the Nash allocation for that battlefield is already substantially higher than the allocation to the other battlefields. Finally, we explore the robustness of our results supporting the salient target hypothesis along two dimensions. The first dimension deals with a lower asymmetric value and its implication that, under the salient target hypothesis, we should find - and actually find - a lower allocation than in the Nash equi-

\footnotetext{
${ }^{7}$ Aberrations from the normative recommendations are explained by behavioural anomalies. A subset of these may disappear with a more controlled design (e.g., order effects due to the graphical representation of the battlefields) while others represent a minor but intrinsic tendency (e.g., natural inclination to choose round numbers). Our experimental design provides a tighter test removing successfully the first subset of anomalies and controlling for the second.
} 
librium prediction. In the second dimension, we replicate the main treatments but include an asymmetry in budgets. High and low budget subjects follow the same pattern of allocations as subject pairs with symmetric budgets. Allocations are consistent with Nash equilibrium in the case of symmetric battlefields but not with asymmetric battlefields; and the deviation from equilibrium is more pronounced in the case of asymmetric values. Thus, the major results in the main treatments appear robust to an asymmetry in budgets.

Our results also provide further evidence of the power of salience outside of the restricted domain of coordination games. Schelling (1960) explains successful coordination in games with multiple equilibria by conjecturing that players beliefs go through a process of "mutual accommodation" anchored by the focality of some equilibria (e.g., Grand Central Station seems a natural place to meet in New York). This mutual accommodation is partially supported by experimental evidence (see Mehta et al., 1994; Bardsley et al., 2010; Crawford et al., 2008; Dugar and Shahriar, 2012; Isoni et al., 2013). Schelling (1960, p.84) claims that a lack of common interest should prevent such mutual accommodation in games of pure conflict. But the empirical question of whether salience actually has an impact in pure conflict games has received limited attention, and exclusively in the context of simple hide-and-seek games (Rubinstein and Tversky, 1993; Rubinstein et al., 1996; Rubinstein, 1999; Hargreaves Heap et al., 2014). Unfortunately, the evidence from this game may not adequately address the empirical question, as there is some controversy about the ability of subjects to follow equilibrium recommendations in such games even when there is no salience (PalaciosHuerta and Volij, 2008; Levitt et al., 2010; Wooders, 2010). The evidence in Colonel Blotto games appears to support equilibrium behavior in games without salience, providing a solid baseline for analysis. Minor deviations from this baseline have previously been rationalized in terms of salience. For instance, salience has been invoked to explain a preponderance of allocations to battlefields that are multiples of ten (Arad and Rubinstein, 2012) and a higher allocation to far left-hand battlefields (Chowdhury et al., 2013). We perform a controlled examination of salience as an explanation for deviations from Nash behavior in Colonel Blotto games and, as in the literature on coordination games and hide-and-seek games, we control ex-ante for different aspects of salience (see footnote 7). This article does so with respect to labels and values, under both symmetric and asymmetric budgets.

The rest of the paper is organized as follows. Section 2 presents the basic model examined and the underlying theoretical predictions. Section 3 describes the experimental design and concrete hypotheses; and section 4 presents the results. Section 5 discusses implications for the examination of multi-battle contests and concludes. 


\section{Basic model and theoretical predictions}

The Colonel Blotto game is a two-player constant-sum game. Two players, $A$ and $B$, simultaneously allocate their corresponding budget $X_{A}$ and $X_{B}$ of a continuous (sunk) resource across the $n$ independent battlefields, such that the allocation to each battlefield is non-negative. Any part of the budget not allocated is lost, so we will assume that each player $i$ 's pure strategy space is the set of non-negative $n$-tuples $x_{i}=\left(x_{i 1}, x_{i 2}, \ldots, x_{i n}\right)$ satisfying $\sum_{j=1}^{n} x_{i j}=X_{i}$. For the purposes of our experiment we set $n=4$ and assume that each battlefield $j$ has a symmetric value across players $v_{j}$ that may differ across battlefields (see Figure 1). ${ }^{8}$ Each player's objective is to maximize the sum of the values of battlefields won. The winner in

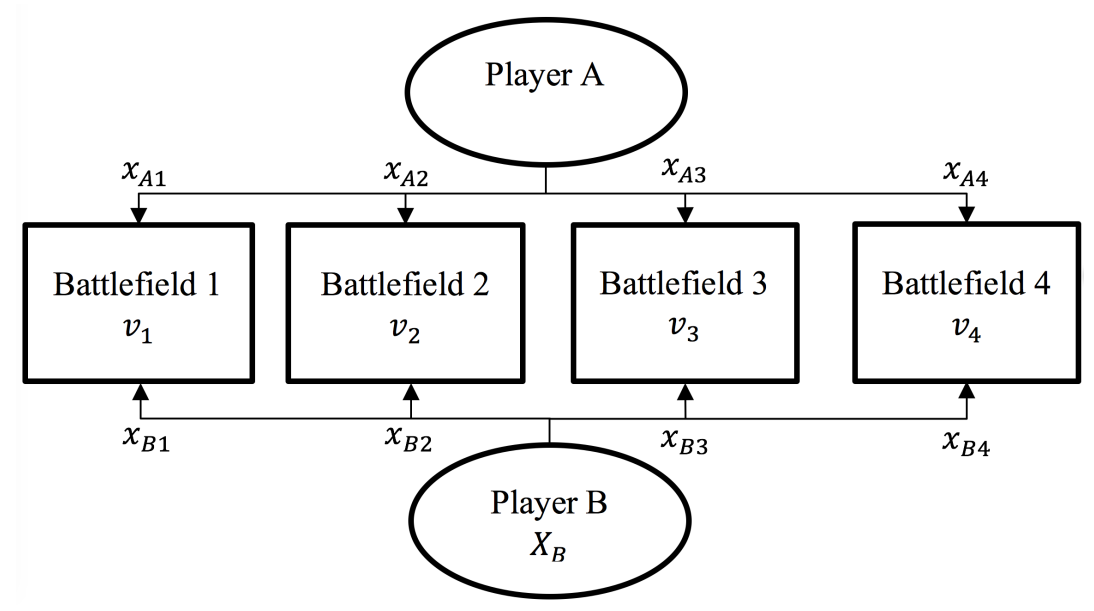

Figure 1: Colonel Blotto game

each battlefield $j$ is determined by a lottery contest success function (Tullock, 1980) and, in the event that both players $i=A, B$ allocate $x_{i j}=0$ to a battlefield, a random draw determines the winner:

$$
p_{i j}\left(x_{i j}, x_{-i j}\right)= \begin{cases}\frac{x_{i j}}{\left(x_{i j}+x_{-i j}\right)} & \text { if } x_{i j}+x_{-i j} \neq 0 \\ \frac{1}{2} & \text { if } x_{i j}=x_{-i j}=0\end{cases}
$$

The solution to this problem can be found in a result due to Friedman (1958).

\footnotetext{
${ }^{8}$ We chose $n=4$ because it is the smallest number of battlefields that allows us to define the salience of a single battlefield with respect to its larger complement within the set of battlefields and at the same time assures that there is one non-salient battlefield that is interior whenever the salient battlefield is interior in the horizontal positioning of battlefields illustrated in Figure 1. (The former requirement rules out $n=2$ and the latter rules out $n=3$.) Both Arad and Rubinstein (2012) and Chowdhury et al. (2013) raise the possibility that allocations may be influenced by the horizontal positioning of battlefields.
} 
Theorem. The pair of $n$-tuples $x_{A}^{*}$ and $x_{B}^{*}$ is a Nash equilibrium of the Colonel Blotto game with a linear count objective, battlefield valuations $\left\{v_{j}\right\}_{j=1}^{n}$ symmetric across players, budget-constrained use-it-or-lose-it costs with budget constraints $X_{A}$ and $X_{B}$, and a lottery CSF in each battlefield, if $\forall j \quad x_{A j}^{*}=X_{A} \frac{v_{j}}{\sum_{k=1}^{n} v_{k}}$ and $x_{B j}^{*}=X_{B} \frac{v_{j}}{\sum_{k=1}^{n} v_{k}}$. The equilibrium expected payoff of player $i$ is $\frac{X_{i}}{X_{A}+X_{B}} \sum_{j=1}^{n} v_{j}$.

For the purposes of this study, two special cases of this theorem are especially relevant. In the case where battlefields are symmetric in value, so that $v_{j}=v$ for every $j$, the optimal allocation across the four battlefields is to set $x_{i j}=X_{i} \frac{v}{4 v}=\frac{X_{i}}{4}$. That is, the optimal allocation requires that each player spread his budget equally across the battlefields. In the case where all battlefields have identical values except battlefield $j$, the optimal allocation of player $i$ to battlefields $j$ and $m \neq j$ are $x_{i j}=X_{i} \frac{v_{j}}{V}$ and $x_{i m}=X_{i} \frac{v_{m}}{V}$, where $V=\sum_{k=1}^{4} v_{k}$. If the value of battlefield $j$ is higher, $\frac{x_{i j}}{x_{i m}}=\frac{v_{j}}{v_{m}}>1$, so that battlefield $j$ receives a proportionally higher allocation than the common allocation to the other battlefields. Alternatively, if the value of battlefield $j$ is lower, battlefield $j$ receives a proportionally lower allocation than the common allocation to the other battlefields.

\subsection{Robustness of predictions to out-of-equilibrium behavior}

It is also of interest in the examination of our experimental data to understand the optimal allocation of player $i, x_{i}=\left(x_{i 1}, \ldots, x_{i n}\right)$, in the event that player $-i$ chooses, or is expected to choose, an allocation, $x_{-i}=\left(x_{-i 1}, \ldots, x_{-i n}\right)$, that is not an equilibrium allocation. Friedman (1958, p.703) demonstrates that, for each feasible vector of allocations of player $-i, x_{-i} \gg 0$, player $i$ 's best response to $x_{-i}$ is to set for each battlefield $j=1, \ldots, n$.

$$
x_{i j}=r_{i j}\left(x_{-i}\right) \equiv \frac{\left(x_{-i j} v_{j}\right)^{\frac{1}{2}}}{\sum_{k=1}^{n}\left(x_{-i k} v_{k}\right)^{\frac{1}{2}}}\left[X_{i}+X_{-i}\right]-x_{-i j}
$$

Note that player $i$ 's best response has the property that the best response in battlefield $j$ to the $n$-tuple $x_{-i}$ depends on the allocations of player $-i$ to battlefields other than battlefield $j$. This arises because the opportunity cost of a unit allocated by player $i$ to battlefield $j$ depends upon $-i$ 's allocations to the other battlefields.

In understanding out of equilibrium behavior in this game, one special case of the rival player - $i$ 's allocation that is of specific interest for illustrative purposes is that in which the allocation of $-i$ to battlefield $j$, $x_{-i j}$, varies, holding the other $(n-1)$ battlefield allocations equal to $\frac{1}{n-1}$ of the remaining budget. In this case, for a given $x_{-i j}$ and $x_{-i k}=\frac{X_{-i}-x_{-i j}}{(n-1)}$ for all $k \neq j$, equation (1) above may be written as

$$
x_{i j}=\tilde{r}_{i j}\left(x_{-i j}\right) \equiv \frac{\left(x_{-i j} v_{j}\right)^{\frac{1}{2}}}{\left(x_{-i j} v_{j}\right)^{\frac{1}{2}}+(n-1)\left[v_{k}\left(\frac{X_{-i-x_{-i j}}}{n-1}\right)\right]^{\frac{1}{2}}}\left[X_{i}+X_{-i}\right]-x_{-i j}
$$

This formulation of the best response of player $i$ in battlefield $j$, obtained by equating player $-i$ 's allocations to all battlefields $k \neq j$, yields $x_{i j}$ as a function of $x_{-i j}$ and the parameters of the game $X_{A}, X_{B}$, 
and $\left(v_{1}, \ldots, v_{n}\right)$. We refer to the function $\tilde{r}_{i j}\left(x_{-i j}\right)$ as $i$ 's conditional best response to $x_{-i j}$ in battlefield $j$. Two combinations of parameters are relevant for our main experimental design. Both involve $n=4$ and $X_{A}=X_{B}=200$. The first combination, that of symmetric valuations, has $v_{k}=15$ for every $k$. The other combination, that of asymmetric valuations, sets the value of the salient battlefield $v_{j}=16.5$ and the remaining battlefields $v_{k}=14.5, k \neq j$.

Figures $2 \mathrm{a}$ and $2 \mathrm{~b}$ below indicate these two conditional best response functions for the symmetric case and asymmetric case with a single high value battlefield. ${ }^{9}$ For both configurations of valuations, $\tilde{r}_{i j}\left(x_{-i j}\right)$ is initially increasing in $x_{-i j}$ (holding $-i$ 's allocations to the remaining battlefields equal) up to the equilibrium levels of $x_{-i j}$ and $x_{i j}$ for the corresponding parameters. In the symmetric case, this is $x_{-i j}=x_{i j}=50$ and, in the asymmetric case, this is $x_{-i j}=x_{i j}=55$. After these values of $x_{-i j}$, player $i^{\prime}$ s conditional best response to $x_{-i j}$ decreases slowly in both configurations until $x_{-i j}$ reaches approximately 118.1 (at which the optimal response $\tilde{r}_{i j}\left(x_{-i j}\right)$ is approximately 45.675$)$ in the symmetric case and 116 (at which the optimal response $\tilde{r}_{i j}\left(x_{-i j}\right)$ is approximately 51.947$)$ in the asymmetric case. For larger values of $x_{-i j}, \tilde{r}_{i j}\left(x_{-i j}\right)$ increases in $x_{-i j}$, approaching 200 as $x_{-i j}$ approaches the complete budget $X_{-i}=200$. For values of $x_{-i j}$ close to 200, player $-i$ is placing so little on the other battlefields $k \neq j$ that player $i$ is able to win those battlefields with near certainty with a very small allocation. Consequently, his optimal response is to place almost all of his budget in battlefield $j$.

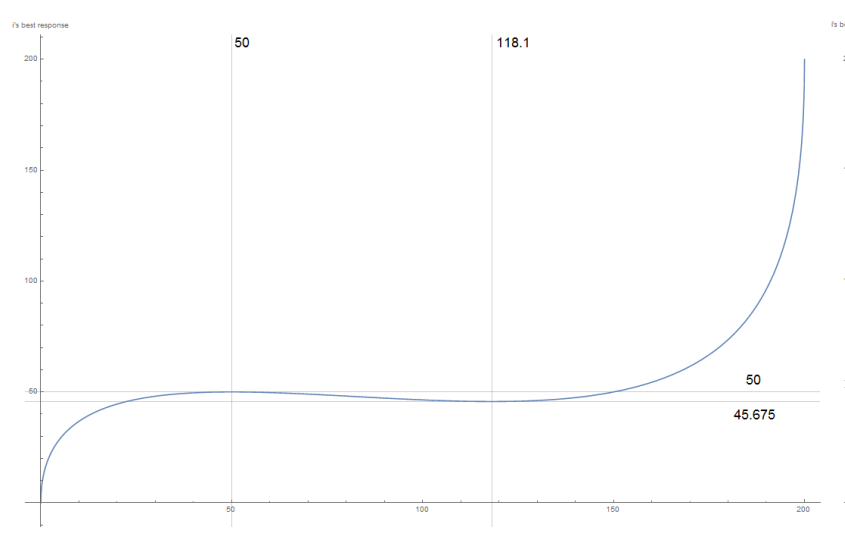

(a) Symmetric case

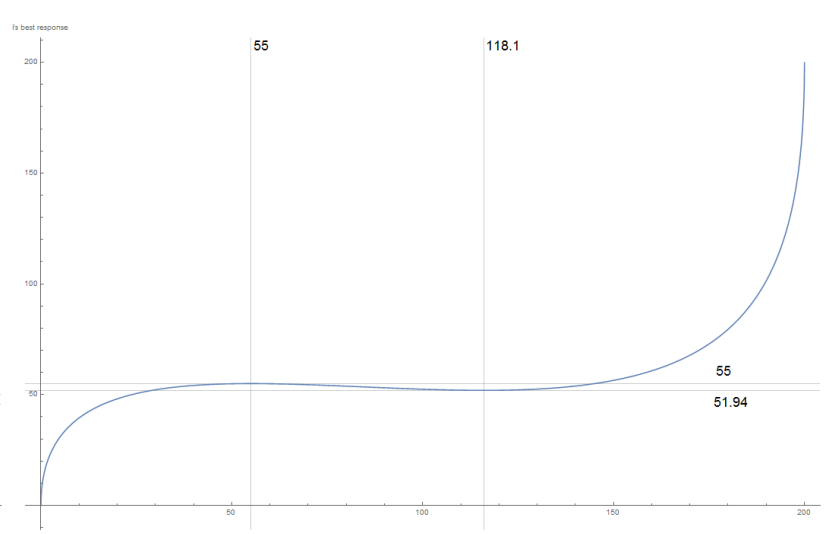

(b) Asymmetric case

Figure 2: Best response functions

An important implication of this analysis of out-of-equilibrium behavior is that the optimal allocation of player $i$ to battlefield $j$ depends on the complete vector of battlefield allocations of the rival player, $x_{-i}$. However, if as $x_{-i j}$ changes player $-i$ maintains identical allocations across all battlefields other than battlefield $j$ (so that $-i$ 's total expenditure equals $X_{-i}$ ), we may examine the optimal response $\tilde{r}_{i j}\left(x_{-i j}\right)$ of

\footnotetext{
${ }^{9}$ The asymmetric case with a low value battlefield can be found in Appendix A.
} 
player $i$ in battlefield $j$ as a function of $x_{-i j}$ alone. From (2), deviations of $x_{-i j}$ away from the equilibrium level, but still in a relatively large neighborhood about the equilibrium level $\left(x_{-i j} \in(0,118.1)\right.$ in the symmetric case and $x_{-i j} \in(0,116)$ in the asymmetric case) lead to a reduction in the optimal response $\tilde{r}_{i j}\left(x_{-i j}\right)$. As a consequence, a player anticipating a rival's over or under allocation to a battlefield relative to equilibrium (due, perhaps, to the salience of that battlefield) will best respond by reducing his own allocation, albeit possibly very slightly. An increase in the allocation $x_{i j}$ is not a best response to a deviation from the equilibrium level of $x_{-i j}$ for a substantial neighborhood about the equilibrium level of $x_{-i j} .{ }^{10}$

Finally, because equilibrium allocations are proportional to a player's budget, in the remainder of the paper, equilibrium and observed allocations are normalized with respect to the player's budget, so that we will refer to the share of a players budget allocated to a battlefield. The allocation share of player $i \in\{A, B\}$ to battlefield $j \in\{1,2,3,4\}$ is defined as $s_{i j}=\frac{x_{i j}}{X_{i}}$.

\section{Experimental design and hypotheses}

\subsection{Experimental design}

We have three main treatments: the symmetric treatment, the focal treatment and the asymmetric treatment. In the symmetric treatment, subjects play 20 periods of a Colonel Blotto game with the same parameters throughout the session. Both subjects have a budget of $X_{i}=200$ tokens that they are required to allocate across the different battlefields. ${ }^{11}$ The four battlefields are represented by a row of four boxes, each containing a white circle. Every box is valued at $v_{j}=15$ points.

The other two treatments present alternative methods to single out one box (the target box) from the others. In the focal treatment, the game is identical to the symmetric treatment except that a label asymmetry is

\footnotetext{
${ }^{10}$ Our discussion of player $i$ 's optimal response to out of equilibrium behavior by player $-i$ in a salient battlefield $j$ assumes that in increasing $x_{-i j}$ above its equilibrium level, the allocations $x_{-i k}, k \neq j$, are reduced symmetrically. Similarly, in decreasing $x_{-i j}$ below its equilibrium level, the $x_{-i k}, k \neq j$, are increased symmetrically. Due to our symmetric treatment of the non-salient battlefields, this seems a reasonable benchmark. However, note that in the non-salient battlefields $k \neq j, v_{k}$ is constant and the allocations $x_{-i k}$, $k \neq j$, appear in the best response function $x_{i j}=r_{i j}\left(x_{-i}\right)$ in (1) only in the denominator, where $\left(x_{-i k}\right)^{1 / 2}$ is concave in $x_{-i k}$. As a result, for a fixed $x_{-i j}$, from Jensens inequality, the denominator in (1) is maximized over all $x_{-i k}, k \neq j$, satisfying player $-i$ 's budget constraint by setting $x_{-i k}=\frac{X_{-i}-x_{-i j}}{3}$. Consequently, setting $x_{-i k}=\frac{X_{-i}-x_{-i j}}{3}$ minimizes the value of $x_{i j}=r_{i j}\left(x_{-i}\right)$ for a fixed value of $x_{-i j}$. Nonetheless, it is possible to show that if one perturbs $x_{-i}$ in a neighborhood of its equilibrium level by raising the allocation to the salient battlefield $x_{-i j}$ and symmetrically reducing the allocations of only a subset of the remaining battlefields (while holding the allocations to the other battlefields constant at the equilibrium levels) one obtains a qualitatively similar results - player $i$ 's optimal response to the deviation from equilibrium behavior is to reduce his allocation $x_{i j}=r_{i j}\left(x_{-i}\right)$. This includes the extreme case, in which the allocation to only one of the battlefields $k \neq j$ is reduced with the increase in $x_{-i j}$ (while the others at kept constant at the equilibrium level). Similarly, if one perturbs $x_{-i}$ in a neighborhood of its equilibrium level by lowering the allocation to the salient battlefield $x_{-i j}$ and symmetrically increasing the allocations of only a subset of the remaining battlefields (while holding the allocations to the other battlefields constant at the equilibrium levels), player $i$ 's optimal response to the deviation from equilibrium behavior is again to reduce his allocation $x_{i j}=r_{i j}\left(x_{-i}\right)$. Consequently, the best response behavior of player $i$ to a range of deviations from the equilibrium that involve misallocation of $-i$ to the salient battlefield $j$ and misallocation in (weakly) the opposite direction for battlefields $k \neq j$ yields a reduction of $i^{\prime}$ s allocation $x_{i j}$ to the salient battlefield $j$. It is, however, possible to obtain an increase in the best response $x_{i j}=r_{i j}\left(x_{-i}\right)$ to a perturbation of $x_{-i}$ in a neighborhood of its equilibrium level if an increase in $x_{-i j}$ is accompanied by an increase in $x_{-i k}$, for some $k \neq j$.

${ }^{11}$ Because we employ a lottery CSF and budgets are use-it-or-lose-it, any optimal allocation must exhaust the budget. In addition, continuity in the space of allocations is approximated by allowing subjects to include one decimal in their allocations. Actually, only $5.71 \%$ of all allocation decisions included decimals.
} 
added: one box contains a black circle and the remaining boxes a white circle. Thus, we induce the salience of a target while maintaining minimal asymmetry to test the salient target hypothesis. In the asymmetric treatment, the game is identical to the symmetric treatment but a value asymmetry is induced: one box is assigned a value of $v_{T}=16.5$ points while the others are valued at $v_{-T}=14.5$ points. Although there is no compelling reason why one specific asymmetric configuration of values should be selected, we chose these specific values because they represent the (common) subjective values of each battlefield that are implied if the mean bid across all subjects in the focal treatment is generated by equilibrium behavior. That is, subjects conforming to equilibrium behavior in the asymmetric treatment generate the same mean bid in each battlefield as arises in the data in the focal treatment.

An inherent characteristic of multi-battle contests with a fixed budget is the negative correlation between a players allocations to individual battlefields. In contrast, most attempts to analyze data in multi-battle experimental contests have implicitly assumed independence between battlefields. ${ }^{12}$ To deal with the dependence of allocations arising from the budget constraint, we collapse all of the information concerning a subject's allocation into one single number: the allocation share to the target box $\left(s_{i T}\right)$. To justify this approach, note that our games have the following features. First, subjects can only distinguish the boxes either by position or by being the target box. ${ }^{13}$ Second, the positions of the boxes are randomized across pairs every period; and subjects are informed of this. This randomization allows potential position effects of the boxes to be controlled. If the position of the box has no significant effect, the implication is that subjects can only distinguish the target box from the others. In that case, all relevant information about a subject's allocation is divided into the allocation share to the target box $\left(s_{i T}\right)$ and the remaining joint share that the other boxes receive $1-s_{i T}$. If successful, this method addresses the concern about dependence in our data. In section 4.1., we report the absence of evidence for position effects and proceed under the understanding that the relevant information to test our hypotheses resides in the allocation share to the target box.

In addition, we design four other treatments to check for the validity and robustness of our results. First, the motivating applications in the introduction suggest a positive relationship between salience and allocations to the target box. For an increased external validity of the experiment, the parameters in the main treatments are selected to be informative about the potential positive relationship between salience and allocations. However, salience can also affect allocations negatively. For example, in a game with a target box valued at 13.5 and others at 15.5 , we should expect that, contrary to the case with a single high value, salience will induce negative psychological payoffs and, therefore, an under-allocation to the target box. We investigate this game in our low value asymmetry treatment. Second, we examine the same three main treatments implementing a budget asymmetry: one subject (the strong player) receives a budget of $X_{s}=200$ tokens and the other one (the weak player) receives a budget of $X_{w}=160$ tokens. Thus, we have 7 treatments. Each treatment is represented by a two letter acronym. The first letter indicates whether budgets

\footnotetext{
${ }^{12}$ An exception is Arad and Rubinstein (2012), who present exclusively summary statistics.

${ }^{13}$ The experimenter, however, can identify each box by an ID number from 1 to 4 . The box with ID 1 is always the target box in each treatment (even in the symmetric treatment where boxes cannot be distinguished otherwise).
} 
are identical (I) or asymmetric (A) and the second letter indicates whether boxes are symmetric (S), focal (F) or asymmetric in values - with the case in which the target has higher value labeled (V) and the case in which it is of low value labeled (L). For example, IF means identical budgets and a focal box. For treatments with identical (asymmetric) budgets, we run two (four) sessions with a total of 32 subjects with 200 tokens (and 32 subjects with 160 tokens) - see Table 1 for a summary. Each subject is allowed to take part

Table 1: Experimental design

\begin{tabular}{cccccc}
\hline Treatment & Budget (P1; P2) & Label (target; others) & Value (target; others) & \# Sessions & \# Subjects \\
\hline \multicolumn{7}{c}{ Main treatments } \\
IS & $(200 ; 200)$ & (White; White) & $(15 ; 15)$ & 2 & 32 \\
IF & $(200 ; 200)$ & (Black; White) & $(15 ; 15)$ & 2 & 32 \\
IV & $(200 ; 200)$ & (White; White) & $(16.5 ; 14.5)$ & 2 & 32 \\
\multicolumn{7}{c}{ Robustness checks } \\
IL & $(200 ; 200)$ & (White; White) & $(13.5 ; 15.5)$ & 2 & 32 \\
AS & $(200 ; 160)$ & (White; White) & $(15 ; 15)$ & 4 & 64 \\
AF & $(200 ; 160)$ & (Black; White) & $(15 ; 15)$ & 4 & 64 \\
AV & $(200 ; 160)$ & (White; White) & $(16.5 ; 14.5)$ & 4 & 64 \\
\hline
\end{tabular}

in only one session. Within a session, two groups are formed; and, in every period, subjects are matched randomly and anonymously with a subject from the other group. Each subject is informed of their own allocations, their opponent's allocations, the winner of each box and earnings at the end of each period. To show how a winner is determined in each battlefield, subjects are shown a roulette wheel divided into two portions corresponding to each player's proportional allocation for that battlefield. An animated spinning arrow determines the winner randomly by stopping in one of the subjects' portions (see the supplementary material for screenshots). At the end of the experiment, subjects receive a payment based on the points they accumulate in five randomly selected periods, with an exchange rate of $\$ 1$ for every 20 points.

The experiment was conducted at the Economic Science Institute Laboratory at Chapman University. Subjects were recruited from the common subject pool - excluding subjects who had previous experience in contest experiments - and participated anonymously at computer workstations. The show-up fee was $\$ 7$. Instructions (reproduced in Appendix B) were presented on a separate piece of paper and subjects were informed that the same instructions were given to every subject in the session. After reading the instructions, subjects were required to complete a quiz (see supplementary material). Sessions lasted for approximately 65 minutes - including 10 minutes for instructions. By design, subjects receive an average earnings of $\$ 14.5$.

\subsection{Hypotheses}

First, we restrict our attention to the treatments with identical budgets. The null hypothesis across treatments is that behavior is consistent with the Nash Equilibrium (NE). The NE prediction is different in games 
with battlefields with symmetric values (treatments IS and IF) and games with a battlefield of asymmetric value (IV and IL). The prediction in the former treatments should not change the optimal allocation of the NE because labels are payoff irrelevant. For the values selected in our experimental design, the null hypothesis of NE produces two statements:

H1 (NE-symmetry): In the IS and IF treatments, the mean share allocated to the target box $\mathrm{T}$ is $\bar{s}_{i T}=0.25$.

In games with asymmetric values, the NE prediction changes slightly:

H2 (NE-asymmetry): In the IV and IL treatments, the mean shares allocated to the target box $T$ are $\bar{s}_{i T}=0.275$ and $\bar{s}_{i T}=0.225$, respectively.

The salient target hypothesis leads to a different behavioral pattern across treatments. In the completely symmetric battlefield case (treatment IS), this hypothesis and Nash behavior should produce the same results, as there is no salient target. In treatments with battlefield asymmetries, however, a difference in behavior should arise according to the salient target hypothesis. Looking at the evidence in hide-andseek games, Crawford and Iriberri (2007) have suggested that the salient connotations of the labels affect psychological payoffs (e.g., a label of a happy face will translate into a positive payoff). In line with that suggestion, we expect that our connotations in the main treatments should induce positive payoffs. As a consequence, subjects should increase correspondingly their allocations in the salient target. Thus, the corresponding alternative prediction is:

H3 (Label salience): In the IF treatment, the mean share allocated to the target box $T$ is $\bar{s}_{i T}>0.25$.

A similar argument implies that positive (psychological) payoffs are derived from winning the target box with the highest value in the IV treatment and, therefore, higher allocations than the NE prediction are expected:

H4 (Value salience): In the IV treatment, the mean share allocated to target box $T$ is $\bar{s}_{i T}>0.275$.

A natural question arises regarding the relative salience of labels and values. The payoff irrelevance of labels, and the payoff relevance of a higher value, implying that the NE allocation is higher in the latter case, suggest that the effect of value salience should be stronger. We examine this empirical question in the next section.

To check how general the salient target hypothesis is, we move beyond the positive relationship between salience and allocations and test if a lower value of the target box will induce under-allocation. A mirror argument of the one leading to $\mathrm{H} 4$ implies that negative (psychological) payoffs are derived from winning the target box with the lowest value in the IL treatment and, therefore, lower allocations than the NE prediction are expected:

H5 (Low value salience): In the IL treatment, the mean share allocated to target box $T$ is $\bar{s}_{i T}<0.225$.

Finally, we check the robustness of our main results by including asymmetric budgets. Determining the predictions for the treatments with a budget asymmetry is straightforward. Because optimal allocation shares 
are invariant to the size of the budgets, each treatment with a budget asymmetry should produce the same NE prediction as its counterpart with identical budgets. Thus, if our results supporting the salient target hypothesis were considered just a spurious coincidence, finding the same results with budget asymmetries would be unlikely. Alternatively, budget asymmetries could induce more awareness about equilibrium recommendations. For example, Avrahami and Kareev (2009, p.948) find that, especially, the weaker players "approximate the sophisticated game-theoretic solution". Given this prior in favor of equilibrium in the presence of budget asymmetries, if we find evidence for the salient target hypothesis in treatments with identical budgets as well as asymmetric budgets, we can claim strong support for our results.

H6 (Robust results): In each of our main treatments, the pattern in the identical budget version (either $\bar{s}_{i T}^{I}=\bar{s}_{N E}$ or $\left.\bar{s}_{i T}^{I}>\bar{s}_{N E}\right)$ should be the same as in the asymmetric budget version $\left(\bar{s}_{i T}^{A}=\bar{s}_{N E}\right.$ or $\left.\bar{s}_{i T}^{A}>\bar{s}_{N E}\right)$, where superscript $I$ and $A$ refer to the identical budget version of the treatment and the asymmetric budget version, respectively, and $\bar{s}_{N E}$ refers to the equilibrium share.

A summary of the predictions for each treatment by the two competing hypotheses is presented in Table 2 . This summary makes clear that our design separates the Nash Equilibrium and the salient target hypothesis.

Table 2: Summary of predictions

\begin{tabular}{r|cccccccc}
\hline & \multicolumn{10}{c}{$\overline{\mathrm{s}}_{\text {iT }}$ in Treatments } \\
\hline Predictions & IS & IF & IV & IL & AS & AF & AV \\
& & & & & & & \\
Nash Equilibrium hypothesis & $=0.25$ & $=0.25$ & $=0.275$ & $=0.225$ & $=0.25$ & $=0.25$ & $=0.275$ \\
Salient Target hypothesis & $=0.25$ & $>0.25$ & $>0.275$ & $<0.225$ & $=0.25$ & $>0.25$ & $>0.275$ \\
\hline
\end{tabular}

\section{Results}

\subsection{Data overview}

Our variable of interest is the within-subject mean share allocated to the target box $\left(s_{i T}\right)$. As noted in section 3.1., the share allocated to the target box is a better measure of the effect of salience if the position of the boxes has no significant effect. A Friedman-test reveals that the individual average allocation is not significantly different across locations ( $p$-value $=1.00$ ). This result is also robust across treatments. To support our claim of lack of position effects and the assumption of equal allocation to the non-salient battlefields in the out-of-equilibrium analysis, we also test whether the distribution of the remaining budget allocates one third to each of the non-salient boxes. One out of 21 Wilcoxon signed rank tests $(7$ treatments $x 3$ nonsalient boxes) appears significant at the $5 \%$ level (the far right box in treatment IV). Table 3 provides the 
corresponding summary statistics of the within-subject mean share allocated to the target box by players with 200 tokens. ${ }^{14}$

Table 3: Summary statistics

\begin{tabular}{rcccccccc}
\hline Statistic & Periods & \multicolumn{7}{c}{ Within-subject mean share } \\
& \multirow{2}{*}{ All } & IS & IF & IV & IL & AS & AF & AV \\
$\mathbf{N}$ & & 32 & 32 & 32 & 32 & 32 & 32 & 32 \\
Mean & & 0.257 & 0.273 & 0.317 & 0.193 & 0.248 & 0.261 & 0.33 \\
std. dev. & & 0.029 & 0.053 & 0.076 & 0.05 & 0.021 & 0.025 & 0.121 \\
$25 \%$ & & 0.244 & 0.244 & 0.269 & 0.159 & 0.238 & 0.242 & 0.276 \\
$50 \%$ (median) & & 0.255 & 0.262 & 0.3 & 0.203 & 0.249 & 0.255 & 0.332 \\
$75 \%$ & & 0.267 & 0.296 & 0.356 & 0.239 & 0.261 & 0.272 & 0.402 \\
& $\mathbf{1 - 1 0}$ & & & & & & & \\
$\mathrm{N}$ & & 32 & 32 & 32 & 32 & 32 & 32 & 32 \\
Mean & & 0.257 & 0.282 & 0.316 & 0.194 & 0.244 & 0.262 & 0.32 \\
std. dev. & & 0.056 & 0.062 & 0.085 & 0.054 & 0.036 & 0.03 & 0.142 \\
$25 \%$ & & 0.219 & 0.244 & 0.26 & 0.15 & 0.222 & 0.243 & 0.269 \\
$50 \%$ (median) & & 0.261 & 0.266 & 0.308 & 0.197 & 0.244 & 0.258 & 0.322 \\
$75 \%$ & & 0.275 & 0.307 & 0.359 & 0.237 & 0.264 & 0.279 & 0.39 \\
& $\mathbf{1 1 - 2 0}$ & & & & & & & \\
$\mathrm{N}$ & & 32 & 32 & 32 & 32 & 32 & 32 & 32 \\
Mean & & 0.256 & 0.264 & 0.317 & 0.191 & 0.252 & 0.26 & 0.339 \\
std. dev. & & 0.027 & 0.063 & 0.086 & 0.073 & 0.025 & 0.036 & 0.115 \\
$25 \%$ & & 0.236 & 0.242 & 0.266 & 0.143 & 0.24 & 0.245 & 0.265 \\
$50 \%$ (median) & & 0.251 & 0.254 & 0.299 & 0.191 & 0.25 & 0.255 & 0.344 \\
$75 \%$ & & 0.275 & 0.286 & 0.372 & 0.235 & 0.263 & 0.27 & 0.404 \\
\hline
\end{tabular}

Unit of observation is the within-subject mean share $s_{t, u}^{i}$ allocated by subject $i$ to the target box over periods $t$ to $u$.

The summary statistics shown in Table 3 reveal sufficient variation across the different treatments that can address our hypotheses. ${ }^{15}$ First, H1, the NE-symmetry prediction, requires that the within-subject means equal 0.25 in treatments IS and IF. This hypothesis seems to receive support in the treatment with completely symmetric battlefields (IS). In the IF treatment, H3, the alternative prediction of label salience, appears to be supported. Second, H2, the NE-asymmetry prediction, seems to be rejected in favor of $\mathrm{H} 4$ and H5, the value salience and the low value salience predictions, respectively. Furthermore, our data suggests that the effect of value salience is higher than that of label salience. All of this suggests support for the salient target hypothesis. Moreover, this pattern appears to be robust in comparing IS to AS, IF to AF, and IV to AV in line with H6. We test H1-H6 formally in section 4.2.

Before proceeding, we address a confounding factor that could potentially affect the interpretation of these tests. By using the within-subject mean share allocation as the unit of observation, we abstract away from potential time trends. The data appear to support this position. For each treatment, we test whether changes

\footnotetext{
${ }^{14}$ Summary statistics in which the unit of observation is the allocation can be found in the supplementary material.

${ }^{15}$ We employ one-way Anova to test the joint null hypothesis that all treatments have the same mean share to the target box. Results show that the averages are not the same across treatments $(1-10$ periods $p$-value: .0001; 11-20 periods $p$-value: $<.0001$; all periods $p$-value: $<.0001)$. Similar results are obtained using a Kruskal-Wallis test to address concerns about normality.
} 
in the within-subject mean and standard deviation between periods 1-10 and 11-20 are different from zero (see Table 4 for the results). The repeated nature of our experiment does not affect the mean allocation share though it reduces the dispersion in most treatments.

Table 4: Individual changes between first and second half of the session

\begin{tabular}{rrrrrrrr}
\hline \multicolumn{1}{c}{ Treatments: } & IS & IF & IV & IL & AS & AF & AV \\
\hline \multicolumn{2}{r}{ change between periods 1-10 and 11-20 } & in means & & & \\
mean & -.0019 & -.019 & 0.001 & -.0033 & 0.008 & -.0027 & 0.0189 \\
median & 0.0005 & -.023 & -.0086 & -.0025 & 0.0112 & 0 & 0.0287 \\
t-test of zero difference & 0.871 & 0.117 & 0.939 & 0.821 & 0.356 & 0.723 & 0.226 \\
signed rank test of zero difference & 0.575 & 0.058 & 0.41 & 0.282 & 0.135 & 0.808 & 0.071
\end{tabular}

change between periods 1-10 and 11-20 in standard deviations

\begin{tabular}{rlllllll} 
mean & -.006 & -.0286 & -.04 & -.0175 & -.017 & -.0076 & -.0164 \\
median & -.002 & -.0122 & -.018 & -.0148 & -.017 & -.013 & -.0149 \\
$t$-test of zero difference & 0.468 & $.04^{* *}$ & $.003^{* * *}$ & $.026^{* *}$ & $.022^{* *}$ & 0.236 & 0.275 \\
signed rank test of zero difference & 0.531 & $.025^{* *}$ & $.007^{* * *}$ & $.029^{* *}$ & $.013^{* *}$ & 0.137 & 0.175 \\
\hline $\begin{array}{l}\text { Note: the values in the cells corresponding to rows of the statistical tests are } \\
\text { p-values. }\end{array}$ \\
respectively.
\end{tabular}

\subsection{Main results}

Figure 3 illustrates the within-subject mean share allocated to the target box by players with a budget of 200 tokens with the corresponding 95\% confidence interval. The formal comparison across treatments can be examined visually.

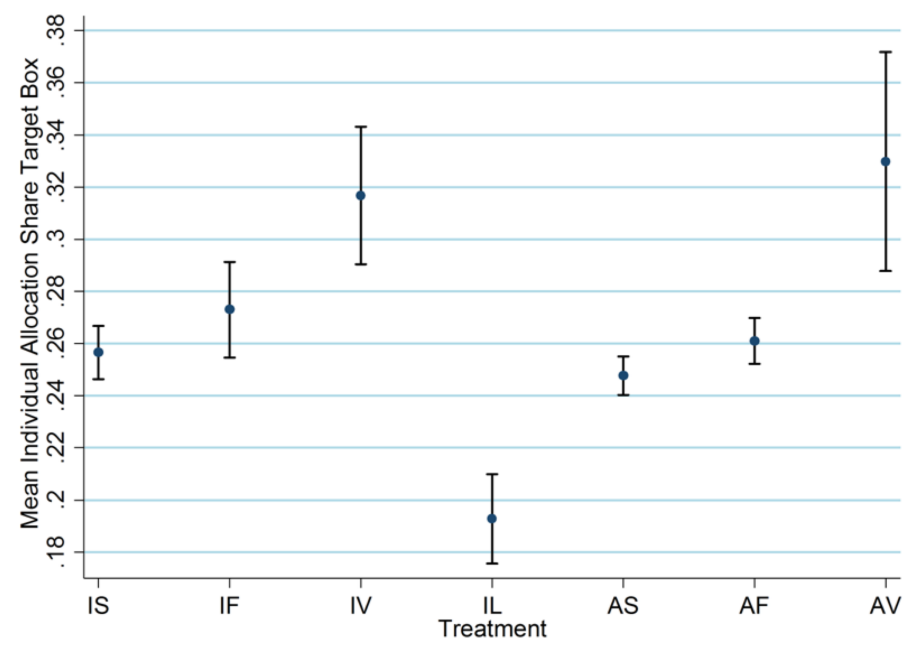

Figure 3: Mean individual allocation share 
First, in the IS and IF treatments, H1, the NE prediction, requires $\bar{s}_{i T}=0.25$. In Figure 3, checking whether 0.25 is within the $95 \%$ confidence interval for the within-subject mean allocation share to the target box in treatments IS and IF, we obtain result 1.

Result 1: In IS, the mean allocation share is consistent with H1, the NE-symmetry prediction. In IF, the mean allocation share is consistent with $\mathrm{H} 3$, the label salience prediction. Therefore, our results in the IS and IF treatments support the salient target hypothesis.

Our results in the IS treatment are reassuring. Because our experimental design controls for some of the sources of minor deviations from equilibrium play in previous studies, ${ }^{16}$ we replicate previous evidence in support of equilibrium play under a more demanding test. In the IF treatment, the Nash equilibrium hypothesis would require again a share of 0.25 in the target box, while the salient target hypothesis requires a finding of label salience. The lower bound of the confidence interval of the IF treatment does not reach 0.25 , consistent with $\mathrm{H} 3$, the label salience prediction. From the perspective of equilibrium play this result is interesting because the game is ex-ante formally the same as in the completely symmetric case. But the salience of the label appears to be sufficient to trigger over-allocation to the target box.

Second, in examining the effect of value asymmetry in the IV treatment, $\mathrm{H} 2$, the NE-asymmetry prediction, requires that 0.275 lie within the $95 \%$ confidence interval of the within-subject mean allocation share to the target box. In contrast, $\mathrm{H} 4$, the value salience prediction, requires that 0.275 lie below the lower bound of the $95 \%$ confidence interval for that treatment. The latter is clearly observed in Figure 3.

Result 2: In IV, the mean allocation share is inconsistent with H2, the NE-asymmetry prediction, and consistent with $\mathrm{H} 4$, the value salience prediction. Therefore, our results in IV support the salient target hypothesis.

Results 1 and 2 suggest that the support for equilibrium found in the literature disappears as soon as small asymmetries are introduced in the boxes through labels (a change of color) or values (a change in 1.5 points: roughly 7 cents on the dollar). Fortunately, deviation from equilibrium behavior occurs in a systematic direction that is predicted by the salient target hypothesis. In addition, our results also suggest that asymmetry in values is more prominent than asymmetry in labels. With asymmetric values, the mean allocation share deviates from the equilibrium prediction by 0.047 , or $17.4 \%$ of the equilibrium level. With asymmetric labels the mean allocation share deviates from the equilibrium prediction by 0.023 , or $9.2 \%$ of the equilibrium level. This result is expected as labels are payoff irrelevant aspects of the game.

We now evaluate the robustness of these results. Although our motivating examples and main treatments focus on the positive relationship between salience and allocations, one implication of the salient target hypothesis is that lower values in the target box should lead to under-allocation. We evaluate this statement

\footnotetext{
${ }^{16}$ Arad and Rubinstein (2012) find that people tend to under-allocate in the first and last battlefields in a row while Chowdhury et al. (2013) find that people tend to over-allocate in the battlefields to the left and under-allocate in the ones to the right.
} 
by comparing the mean share allocated to the target box in IL to the prediction in $\mathrm{H} 2$ - requiring that 0.225 lie within the $95 \%$ confidence interval of the within-subject mean allocation share to the target box. In contrast, $\mathrm{H} 5$ suggests that 0.225 lies above the $95 \%$ confidence interval. This is clearly observed in Figure 3 .

Result 3: In IL, the mean allocation share is inconsistent with H2, the NE-asymmetry prediction, and consistent with H5, the low value salience prediction. Therefore, our results in IL support the salient target hypothesis in both the positive and negative domains of the relationship between salience and allocations.

Finally, we compare the mean share allocated to the target box in treatments with identical budgets and asymmetric budgets. This comparison tests $\mathrm{H6}$, that our results are robust. This comparison is restricted to players with 200 tokens, the only common type between treatments with identical and asymmetric budgets. Before stating the result of this comparison, we report how different the mean share allocated to the target box by weak players, those with 160 tokens in the asymmetric budget treatments, is from that of players with 200 tokens in both the identical and asymmetric budget treatments. We first run t-tests and rank sum tests comparing the within-subject mean allocation share between weak players and players in treatments with identical budgets. The corresponding $p$-values appear in Table 5 . The results show that every $p$-value is higher than 0.10 . Thus, there is no significant difference between the means of weak players and players with a symmetric budget of 200 tokens.

Table 5: p-values tests within-subject mean allocation between symmetric and weak players

\begin{tabular}{crccc}
\hline Treatments & Tests & \multicolumn{3}{c}{ Periods } \\
& & $\mathbf{1 - 1 0}$ & $\mathbf{1 1 - 2 0}$ & All \\
\hline \multirow{2}{*}{ IS } & t-test & 0.46 & 0.66 & 0.38 \\
& rank sum & 0.79 & 0.72 & 0.25 \\
IF & t-test & 0.4 & 0.83 & 0.57 \\
& rank sum & 0.85 & 0.82 & 0.82 \\
IA & t-test & 0.7 & 0.98 & 0.87 \\
& rank sum & 0.44 & 0.74 & 0.6 \\
\hline Unit of observation is the within-subject mean share $\bar{x}_{t, u}^{s}$ allocated by \\
subject s to the target box over periods $t$ to $u$. \\
These tests are based on thirty two observations.
\end{tabular}

More importantly, as the behavior of strong players is a strategic response to the behavior of weak players, we compare weak players to strong players. We perform t-tests and signed rank tests with the null hypothesis that the difference of paired observations is not different from zero. Results in Table 6 show that there is no significant difference. Thus, we are confident that any potential difference in the behavior of subjects with a budget of 200 tokens between treatments with identical budgets and asymmetric budgets is due to the difference in budgets. 
Table 6: Share difference between strong and weak players

\begin{tabular}{lccc}
\hline Periods & $\mathbf{1 - 1 0}$ & $\mathbf{1 1 - 2 0}$ & All \\
\hline Mean & -0.0053 & 0.0073 & 0.00099 \\
Median & -0.0062 & 0.0055 & -0.00047 \\
standard deviation & 0.025 & 0.04 & 0.03 \\
t-test of zero difference $(p$-value) & 0.48 & 0.54 & 0.91 \\
signed rank test of zero difference $(p$-value) & 0.53 & 0.75 & 0.69 \\
\hline Unit of observation is the within-session difference $\Delta \bar{x}_{t, u}=\bar{x}_{t, u}^{\text {strong }}-\bar{x}_{t, u}^{\text {weak }}$, where $\bar{x}_{t, u}^{\text {type }}$ is the mean \\
of the share allocated by all type players to the target box over periods $t$ to $u$ ("first $10^{\prime \prime}=1$ to 10, "last $10^{\prime \prime}$ \\
$=11$ to 20, "all" $=1$ to 20$)$. The within-session differencing will remove a linear treatment effect for each of \\
the three treatments. As there are four sessions per treatment, these tests are based on twelve observations \\
of $\Delta \bar{x}_{t, u}$.
\end{tabular}

In examining the effect of budget asymmetry across treatments, $\mathrm{H} 6$ requires that Results 1 and 2 extend to asymmetric budgets. So, we compare allocations in AS, AF and AV to the Nash predictions in $\mathrm{H} 1$ and $\mathrm{H} 2$. The same patterns arise in the asymmetric condition, as shown in Figure 3.

Result 4: In AS , AF, and AV the same pattern arises as in Results 1 and 2: 0.25 and 0.275 lie below the confidence interval of the mean allocation share in $\mathrm{AF}$ and $\mathrm{AV}$, respectively; whereas, 0.25 lies within the confidence interval in AS. This is consistent with H6.

This indicates that our Results 1 and 2 supporting the salient target hypothesis are robust.

\section{Discussion}

Our experiment provides the first controlled evidence for the salient target hypothesis, which asserts that the strategic allocation of a players budget in a multi-battle contest is influenced by salient battlefields. In our experiment, salience arises from a particular asymmetry of one of the battlefields with respect to the others. We examine asymmetries of values and labels. Our results suggest that saliently labeled and high value battlefields receive higher allocations in comparison to both the Nash equilibrium prediction and the case in which labels and values are symmetric. This over-allocation is larger when the asymmetry involves a higher value.

One implication of this result is that it suggests a reinterpretation of recent experimental evidence on Colonel Blotto games providing partial support for Nash equilibrium behavior. Previous studies have indicated that positional effects may lead to small deviations from equilibrium play. Our design controls for positional effects and equilibrium behavior in fact arises in the symmetric case. In stark contrast, subjects deviate from equilibrium behavior in the presence of asymmetries in battlefields. More concretely, subjects over-allocate to saliently labeled or high value battlefields. Duffy and Matros (2013) also study Colonel Blotto games with a lottery contest success function and heterogeneous battlefield values, but where all 
battlefields have different values. In their treatment in which subjects maximize the sum of values of battlefields won, they claim qualitative, but not quantitative, support for the equilibrium prediction. ${ }^{17}$ Qualitative support is equated with higher valued battlefields receiving higher allocation shares or, more formally, the coincidence of the ranks of battlefield values and allocation shares. Our results also preserve the identity of battlefield value and share rankings. However, qualitative support for equilibrium behavior is difficult to reconcile in light of our results on label asymmetry. These results suggest an unambiguous quantitative and qualitative rejection of equilibrium behavior in the presence of a salient battlefield. ${ }^{18}$

To rationalize our result of over-allocation to the salient battlefield as a best-response to an opponent's anticipated or actual allocation, one would have to expect the opponent to allocate an implausibly large share of his budget to the salient battlefield. In particular, assuming symmetric allocations across the nonsalient battlefields (as suggested by the experimental evidence that subjects do not distinguish positions), the realized mean share of at least 0.275 in the IF treatment is supported only with the expectation that the opponent places at least slightly above 0.8 of his budget in the salient battlefield (see Figure 2). Similarly, in the IV treatment, the realized mean share of at least 0.31 is supported only with the expectation that the opponent places at least slightly above 0.82 of his budget in the salient battlefield. In our data, less than $0.5 \%$ of the observations are consistent with this possibility in both treatments. And, feedback between games should provide enough information for subjects to adjust their allocations according to best responses. This does not appear to be the case. This apparent inconsistency between observed behavior and best responses in treatments with salience but support for equilibrium behavior in treatments without salience is a novel result. Previously reported evidence, in hide-and-seek games with salience, did not support equilibrium but appeared consistent with best responses (Rubinstein and Tversky, 1993; Crawford and Iriberri, 2007). This contrast suggests that our evidence makes a stronger case for the role of salience outside of the narrow confines of coordination games. Subjects in our experiment over-allocate to saliently labeled and high value battlefields, while both hiders and seekers choose non-salient options. In our treatment with a unique battlefield with a lower value subjects under-allocate to the low value battlefield.

The results of our laboratory experiments supporting the salient target hypothesis suggest some lessons for the field. First, for the given objective functions and contest success functions examined, when salience is induced through labels or a higher value there is a tendency for the opponent to over allocate resources to the salient target relative to the Nash equilibrium level. The extent that this tendency can be exploited will vary across contexts, but in the models examined here the behavior exhibited would suggest an optimal response that involves reducing the allocation to the salient battlefield below the equilibrium level. Of course, this prescription will generally depend on the nature of the multi-battle contest and may differ when other objective functions and contest success functions are employed. Second, to the extent that

\footnotetext{
${ }^{17}$ Duffy and Matros (2013) also include treatments in which players maximize the probability of winning battlefields yielding more than one-half the total value of all battlefields.

${ }^{18}$ In light of our evidence, a reinterpretation of the Duffy and Matros (2013) data suggests some support for the salient target hypothesis. In their treatment with heterogeneous values and a value maximization objective, subjects appear to over-allocate relative to equilibrium in the two highest valued battlefields and under-allocate in the two lowest valued battlefields.
} 
salience or focality can be manipulated ex ante, there may be a strong incentive to do so. Because the salient targets we have studied in our main treatments attract resources, pre-play expenditures by a player to make an existing target salient may prove useful in causing the rival to sub-optimally divert resources. Again, the extent that this may be exploited will vary with the nature of the multi-battle contest. Exploring these issues under alternative assumptions on contest structure remains a topic for future experimental work. 


\section{References}

ADAMO, T. AND A. MATros (2009): “A Blotto game with incomplete information," Economics Letters, 105, $100-102$.

ARAD, A. (2012): "The tennis coach problem: a game-theoretic and experimental study," The BE Journal of Theoretical Economics, 12, Article 10.

ARAD, A. AND A. Rubinstein (2012): "Multi-dimensional iterative reasoning in action: the case of the Colonel Blotto game," Journal of Economic Behavior \& Organization, 84, 571-585.

Arce, D. G., R. T. Croson, AND C. C. ECKel (2011): “Terrorism experiments," Journal of Peace Research, $48,373-382$.

ARCE, D. G., D. KOVENOCK, AND B. ROBERSON (2012): “Weakest-link attacker-defender games with multiple attack technologies," Naval Research Logistics (NRL), 59, 457-469.

Avrahami, J. AND Y. KAREeV (2009): “Do the weak stand a chance? Distribution of resources in a competitive environment," Cognitive Science, 33, 940-950.

Bachrach, Y., M. Draief, AND S. Goyal (2013): “Contagion and observability in security domains," in Communication, Control, and Computing (Allerton), 2013 51st Annual Allerton Conference on, IEEE, 13641371.

BARdsley, N., J. Mehta, C. StARMeR, AND R. Sugden (2010): “Explaining focal points: cognitive hierarchy theory versus team reasoning," The Economic Journal, 120, 40-79.

BARELli, P., S. Govindan, AND R. WiLsON (2014): “Competition for a majority," Econometrica, 82, 271314.

Bellman, R. (1969): “On "Colonel Blotto” and analogous games," Siam Review, 11, 66-68.

Blackett, D. (1953): “Blotto-type games," in Proceedings Fourth Annual Logistics Conference (Part IIRestricted Session), General Services Administration, Washington DC, 31-41.

BLACKeTt, D. W. (1954): “Some Blotto games,” Naval Research Logistics Quarterly, 1, 55-60.

(1958): "Pure strategy solutions of Blotto games," Naval Research Logistics Quarterly, 5, 107-109.

BOREL, E. (1921): “La theorie du jeu les equations integrales a noyau symetrique. Comptes Rendus del Academie. 173, 1304-1308; English translation by Savage, L. (1953). The theory of play and integral equations with skew symmetric kernels," Econometrica, 21, 97-100. 
BOREL, E. AND J. VILLE (1938): Application de la theorie des probabilities aux jeux de hasard, reprinted in Borel E., Cheron, A.: Theorie mathematique du bridge a la portee de tous, Paris: Gauthier-Villars: Paris: Editions J. Gabay, 1991 ed.

Chia, P. H. And J. ChuAng (2011): "Colonel Blotto in the phishing war," in Decision and Game Theory for Security: Second International Conference, GameSec 2011, College Park, MD, Maryland, USA, November 14-15, 2011, ed. by J. S. Baras, J. Katz, and E. Altman, Springer, 201-218.

ChOWdHury, S. M., D. KOVENOCK, AND R. M. SheremetA (2013): “An experimental investigation of Colonel Blotto games," Economic Theory, 52, 833-861.

CINAR, Y., T. GÖKSEL, ET AL. (2012): “An experimental analysis of Colonel Blotto games under alternative environments," Iktisat Isletme ve Finans, 27, 39-57.

CLARK, D. J. AND K. A. KonRAD (2007): “Asymmetric conflict: Weakest-Link against Best Shot,” Journal of Conflict Resolution, 51, 457-469.

— (2008): “Fragmented property rights and incentives for R\&D," Management Science, 54, 969-981.

Crawford, V. P., U. Gneezy, AND Y. RotTenstreich (2008): "The power of focal points is limited: even minute payoff asymmetry may yield large coordination failures," The American Economic Review, 98, 1443-1458.

CRAWFORD, V. P. AND N. IRIBERRI (2007): "Fatal attraction: salience, naivete, and sophistication in experimental "Hide-and-Seek" games," The American Economic Review, 97, 1731-1750.

CRUTZEN, B. S. AND N. SAHUgUet (2009): “Redistributive politics with distortionary taxation," Journal of Economic Theory, 144, 264-279.

Duffy, J. AND A. MAtros (2013): "Stochastic Asymmetric Blotto Games: Theory and Experimental Evidence," Working Paper 509. University of Pittsburgh, Department of Economics.

DUGAR, S. AND Q. SHAHRIAR (2012): “Focal points and economic efficiency: The role of relative label salience," Southern Economic Journal, 78, 954-975.

DZIUBIŃSKI, M. (2013): “Non-symmetric discrete General Lotto games," International Journal of Game Theory, $42,801-833$.

FRIEDMAN, L. (1958): "Game-theory models in the allocation of advertising expenditures," Operations Research, 6, 699-709.

FUCHS, Z. E. AND P. P. KHARGONEKAR (2012): “A sequential Colonel Blotto game with a sensor network," in American Control Conference (ACC), 2012, IEEE, 1851-1857. 
Golman, R. And S. E. Page (2009): “General Blotto: games of allocative strategic mismatch," Public Choice, 138, 279-299.

Goyal, S. AND A. Vigier (2014): "Attack, defence, and contagion in networks," The Review of Economic Studies, 81, 1518-1542.

Gross, O. AND R. WAGNeR (1950): “A continuous Colonel Blotto game,” Working Paper RM-424. RAND Corporation, Santa Monica.

Gross, O. A. (1950): “The symmetric Blotto game," Working Paper RM-408. Rand Corporation, Santa Monica.

Gupta, A., G. A. Schwartz, C. Langbort, S. S. Sastry, And T. Basar (2014a): “A three-stage Colonel Blotto game: when to provide more information to an adversary in Poovendran R and Saad, W. (eds.) Decision and Game Theory for Security," in American Control Conference (ACC), 2014, GAMESEC 2014, Lecture notes in Computer Science, vol. 8840, 216-233.

- (2014b): "A three-stage Colonel Blotto game with applications to cyberphysical security," in American Control Conference (ACC), 2014, IEEE, 3820-3825.

Hargreaves Heap, S., D. Rojo Arjona, And R. Sugden (2014): “How portable is level-0 behavior? A test of level-k theory in games with non-neutral frames," Econometrica, 82, 1133-1151.

HART, S. (2008): “Discrete Colonel Blotto and general lotto games," International Journal of Game Theory, 36, $441-460$.

- (2015): "Allocation games with caps: from Captain Lotto to all-pay auctions," International Journal of Game Theory, 45, 37-61.

HAUSKEN, K. (2014): "Individual versus overarching protection and attack of assets," Central European Journal of Operations Research, 22, 89-112.

Hernández, D. G. AND D. H. Zanette (2013): "Evolutionary dynamics of resource allocation in the Colonel Blotto game," Journal of Statistical Physics, 151, 623-636.

Hortala-Vallve, R. AND A. Llorente-Saguer (2012): “Pure strategy Nash equilibria in non-zero sum colonel Blotto games," International Journal of Game Theory, 41, 331-343.

IrfanOGLU, Z., S. MAGO, AND R. Sheremeta (2013): “Sequential versus Simultaneous Election Contests: An Experimental Study," Working Paper 14-15. Chapman University, Economic Science Institute.

Isoni, A., A. Poulsen, R. Sugden, AND K. Tsutsui (2013): “Focal points in tacit bargaining problems: Experimental evidence," European Economic Review, 59, 167-188. 
Klumpr, T. And M. K. Polborn (2006): "Primaries and the New Hampshire effect," Journal of Public Economics, 90, 1073-1114.

KOVENOCK, D. AND B. ROBERSON (2008): “Electoral poaching and party identification,” Journal of Theoretical Politics, 20, 275-302.

— (2009a): "Inefficient redistribution and inefficient redistributive politics," Public Choice, 139, 263-272.

—_ (2009b): "Is the 50-state strategy optimal?" Journal of Theoretical Politics, 21, 213-236.

(2011): “A Blotto game with multi-dimensional incomplete information," Economics Letters, 113, 273-275.

(2012a): "Coalitional Colonel Blotto games with application to the economics of alliances," Journal of Public Economic Theory, 14, 653-676.

(2012b): "Conflicts with multiple battlefields," in Oxford Handbook of the Economics of Peace and Conflict, ed. by M. R. Garfinkel and S. Skaperdas, Oxford University Press, 266-290.

(2015): “Generalizations of the General Lotto and Colonel Blotto Games," Working Paper 15-07. Chapman University, Economic Science Institute.

Kovenock, D., B. Roberson, And R. M. Sheremeta (2010): “The attack and defense of weakest-link networks," Working Paper 10-14. Chapman University, Economic Science Institute.

Kvasov, D. (2007): “Contests with limited resources," Journal of Economic Theory, 136, 738-748.

LASLIER, J.-F. (2002): “How two-party competition treats minorities," Review of Economic Design, 7, 297-307.

LevitT, S. D., J. A. List, AND D. H. ReILey (2010): “What happens in the field stays in the field: exploring whether professionals play minimax in laboratory experiments," Econometrica, 78, 1413-1434.

LizZERI, A. AND N. PERSICO (2001): “The provision of public goods under alternative electoral incentives," American Economic Review, 91, 225-239.

MACDONELL, S. T. AND N. MASTRONARDi (2015): “Waging simple wars: a complete characterization of two-battlefield Blotto equilibria," Economic Theory, 58, 183-216.

Mago, S. D. AND R. M. Sheremeta (2012): “Multi-battle contests: an experimental study," Working Paper 12-06. Chapman University, Economic Science Institute.

MCBRIDE, M. AND D. HEWITT (2013): “The enemy you can't see: an investigation of the disruption of dark networks," Journal of Economic Behavior \& Organization, 93, 32-50.

MCDonAlD, J. AND J. TUKey (1949): “Colonel Blotto: a problem of military strategy,” Fortune, 102. 
Mehta, J., C. StARmer, And R. Sugden (1994): “The nature of salience: An experimental investigation of pure coordination games," The American Economic Review, 84, 658-673.

Montero, M., A. Possajennikov, M. Sefton, And T. L. Turocy (2016): “Majoritarian Blotto contests with asymmetric battlefields: an experiment on apex games," Economic Theory, 61, 55-89.

MYERSON, R. B. (1993): "Incentives to cultivate favored minorities under alternative electoral systems." American Political Science Review, 87, 856-869.

Nemeth, S. C. (2010): "A rationalist explanation of terrorist targeting," Ph.D. thesis, University of Iowa.

NikoOfal, M. E. AND J. ZhUANG (2012): "Robust allocation of a defensive budget considering an attacker's private information," Risk Analysis, 32, 930-943.

Osorio, A. (2013): “The lottery Blotto game," Economics Letters, 120, 164-166.

PAlacios-Huerta, I. AND O. VOlij (2008): “Experientia docet: professionals play minimax in laboratory experiments," Econometrica, 76, 71-115.

Powell, R. (2007a): "Allocating defensive resources with private information about vulnerability," American Political Science Review, 101, 799-809.

(2007b): “Defending against terrorist attacks with limited resources," American Political Science Review, 101, 527-541.

(2009): "Sequential, nonzero-sum "Blotto": Allocating defensive resources prior to attack," Games and Economic Behavior, 67, 611-615.

POWERS, M. R. AND Z. SHEN (2009): “Colonel Blotto in the war on terror: implications for event frequency," Journal of Homeland Security and Emergency Management, 6, Article 18.

RietzKe, D. AND B. Roberson (2013): “The robustness of 'enemy-of-my-enemy-is-my-friend'alliances," Social Choice and Welfare, 40, 937-956.

RinOtT, Y., M. SCARSINI, AND Y. YU (2012): “A Colonel Blotto gladiator game," Mathematics of Operations Research, 37, 574-590.

ROBERSON, B. (2006): “The colonel blotto game," Economic Theory, 29, 1-24.

— (2008): "Pork-barrel politics, targetable policies, and fiscal federalism," Journal of the European Economic Association, 6, 819-844.

Roberson, B. AND D. KVAsov (2012): “The non-constant-sum Colonel Blotto game," Economic Theory, 51, 397-433. 
Robson, A. W. (2005): “Multi-item contests,” Working Paper 1885-42587. Australian National University.

RUBINSTEIN, A. (1999): “Experience from a course in game theory: pre-and postclass problem sets as a didactic device," Games and Economic Behavior, 28, 155-170.

RUbinsteIn, A. AND A. TVERSKy (1993): “Naive strategies in zero-sum games,” Working paper 17-93. Tel Aviv University, Sackler Institute of Economic Studies.

Rubinstein, A., A. TVERsky, AND D. Heller (1996): “Naive strategies in competitive games," in Essays in Honor of Reinhard Selten, ed. by P. H. B. M. Wulf Albers, Werner Güth and E. van Damme, SpringerVerlag, 394-402.

SCHELLING, T. C. (1960): The strategy of conflict, Harvard university press.

Schwartz, G., P. Loiseau, AND S. SAStry (2014): “The heterogeneous Colonel Blotto Game," in NETGCOOP 2014, International Conference on Network Games, Control and Optimization, October 29-31.

Sela, A. AND E. EREZ (2013): "Dynamic contests with resource constraints," Social Choice and Welfare, 41, 863-882.

SHUBIK, M. AND R. J. Weber (1981): “Systems defense games: Colonel Blotto, command and control," Naval Research Logistics Quarterly, 28, 281-287.

SNYDER, J. M. (1989): “Election goals and the allocation of campaign resources," Econometrica, 57, 637-660.

Thomas, C. (2012): “N-dimensional Colonel Blotto games with asymmetric valuations. University of Texas," Tech. report. University of Texas.

TULLOCK, G. (1980): “Efficient rent seeking," in Toward a theory of the rent-seeking society, ed. by J. M. Buchanan, R. D. Tollison, and G. Tullock, College Station, TX: Texas A\&M University Press, 97-113.

WASHBURN, A. (2013): “OR Forum-Blotto Politics,” Operations Research, 61, 532-543.

WeINSTEIN, J. (2012): “Two notes on the Blotto game," The BE Journal of Theoretical Economics, 12, Article 7.

WoOders, J. (2010): “Does experience teach? Professionals and minimax play in the lab," Econometrica, 78, $1143-1154$ 


\section{Appendices}

\section{Appendix A: Out of equilibrium behavior with a single low value battlefield}

As in the other two cases, the combination of parameters involves $n=4$ and $X_{A}=X_{B}=200$, but sets the value of the salient battlefield $v_{j}=13.5$ and the remaining battlefields $v_{k}=15.5, k \neq j$. Figures A1 below indicate the corresponding conditional best response function. For this configuration of valuations, $\tilde{r}_{i j}\left(x_{-i j}\right)$ is initially increasing in $x_{-i j}$ (holding $-i$ 's allocations to the remaining battlefields equal) up to the equilibrium levels $x_{-i j}=x_{i j}=45$. After these values of $x_{-i j}$, player $i$ 's conditional best response to $x_{-i j}$ decreases slowly until $x_{-i j}$ reaches approximately 120.3 (at which the optimal response $\tilde{r}_{i j}\left(x_{-i j}\right)$ is approximately 39.023). For larger values of $x_{-i j}, \tilde{r}_{i j}\left(x_{-i j}\right)$ increases in $x_{-i j}$, approaching 200 as $x_{-i j}$ approaches the complete budget $X_{-i}=200$. For values of $x_{-i j}$ close to 200, player $-i$ is placing so little on the other battlefields $k \neq j$ that player $i$ is able to win those battlefields with near certainty with a very small allocation. Consequently, his optimal response is to place almost all of this budget in battlefield $j$.

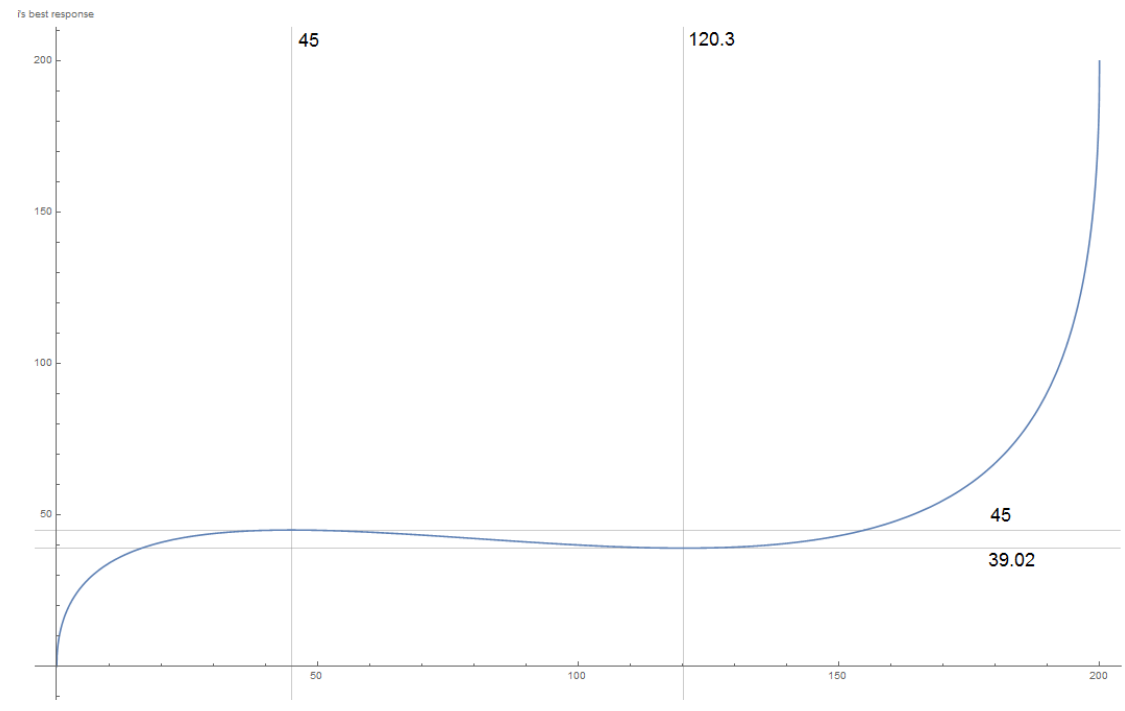

Figure A1: Best response

The function follows a pattern similar to the symmetric and asymmetric high value case. Thus, a player should under-allocate as a response to other players under-allocating. The average allocation to the target box in our IL treatment is 38.55 . This represents the best response to a common opponent's allocation of 16.5 to the target box. It appears unlikely that this accurately reflects the expectations of opponent behavior, as only $2 \%$ of the observations are consistent with this possibility. 


\section{Appendix B: Instructions}

The instructions below were distributed in the completely symmetric case (IS). Instructions for other treatments are just variations highlighted between brackets in the text.

\section{GENERAL INSTRUCTIONS}

Thanks for taking part in this experiment.

You are requested not to communicate during the experiment. If you are not sure about what you are being asked to do, raise your hand and the experimenter will come to your desk to help you. You will be paid $\$ 7$ for turning up plus whatever you earn in the experiment. You will be presented with 20 decision problems. At the end of the experiment, five of these problems will be randomly picked and you will be paid accordingly. You will be paid at the end of the experiment in private and in cash.

\section{YOUR DECISION}

You will be assigned as either participant 1 or participant 2 throughout the experiment. In each decision problem, you will be paired with a randomly selected person whose participant number is different from yours. You will never be told who you have been paired with. Everyone in this room is reading the same set of instructions.

In each decision problem, both participants will receive 200 tokens [budget asymmetry treatments: In each decision problem, participant 1 will receive 200 tokens and participant 2 will receive 160 tokens]. Each participant will choose how to allocate their tokens among four boxes. The four boxes are labelled and shown in a row. The position of the boxes is determined randomly and their labels are white circles [focal treatments: The position of the boxes is determined randomly and one box is labelled with a black circle and the others with a white circle]. You and the participant you have been paired with in that decision problem see the same display of boxes.

Each participant must allocate every token before proceeding to the next decision problem. You do that by writing the number of tokens you want to allocate to certain box in the slot below. You can use decimal numbers.

\section{YOUR EARNINGS}

In every decision problem, each box is worth 15 points [high value asymmetry treatments: In every decision problem, one box is worth 16.5 points and the other three 14.5 points each] [low value asymmetry treatments: In every decision problem, one box is worth 13.5 points and the other three 15.5 points each]. If that decision problem is selected at the end of the experiment, you will earn the points of the boxes you win, which will be converted into cash at a rate of $\$ 1$ per 20 points. Your objective is to earn as many points as possible.

How do you win a particular box? 
Your chances of winning a particular box are given by the number of tokens that you allocated divided by the total number of tokens allocated by you and the other participant. In case both participants allocate zero to the same box, each participant has $50 \%$ chance of winning that box. After allocating the tokens, these proportions are shown in a roulette below each box and the computer will spin an arrow. You win if the arrow finishes in your part of the roulette.

After every decision problem, you will see the allocation of your tokens, the allocation of the other participant's tokens, which boxes you win and your possible earnings in that decision problem.

Before starting the experiment, a questionnaire will check that the instructions were clear. Please complete the questionnaire and raise your hand. The experimenter will come to pick it up and clarify any doubt. 


\section{Supplementary material}

\section{Supplementary material 1 [not intended for publication]: Screenshots}

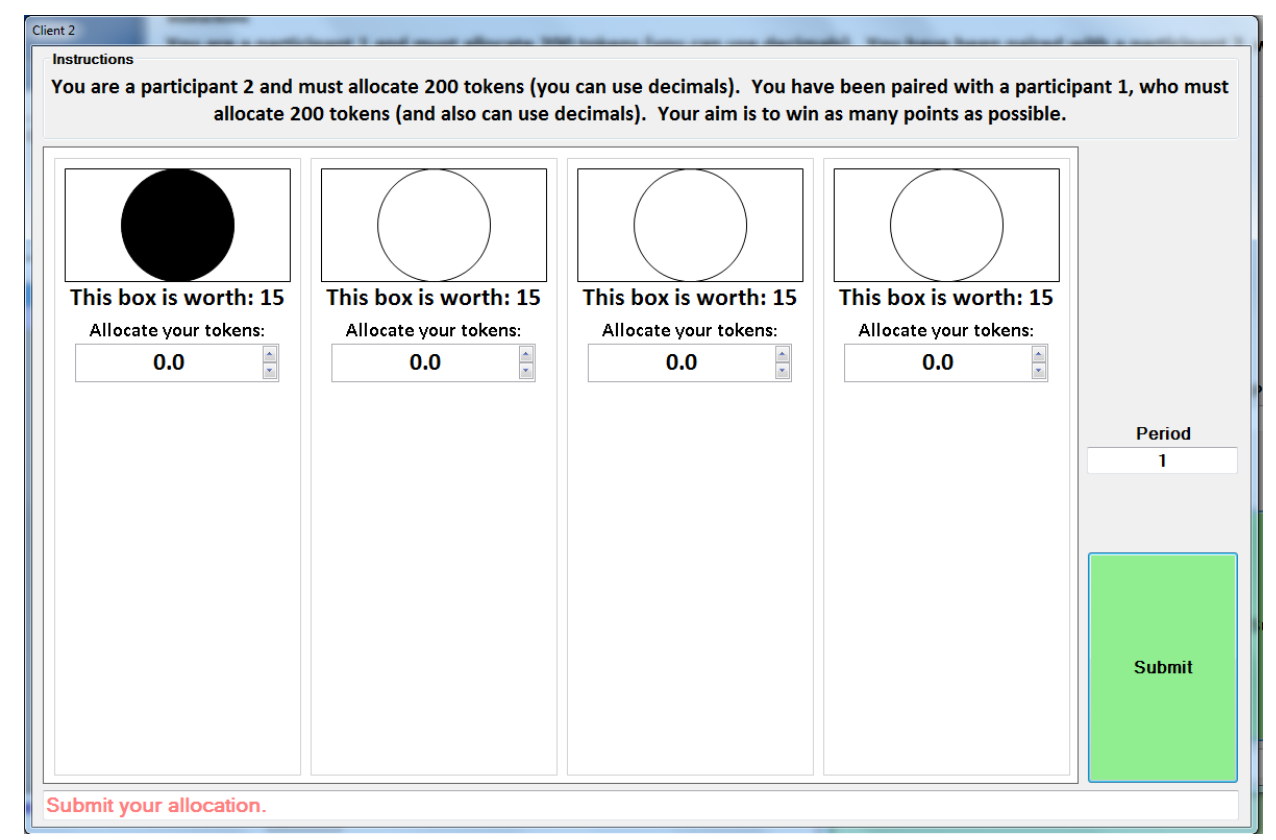

Screenshot 1: Decision screen (example of IF treatment) 


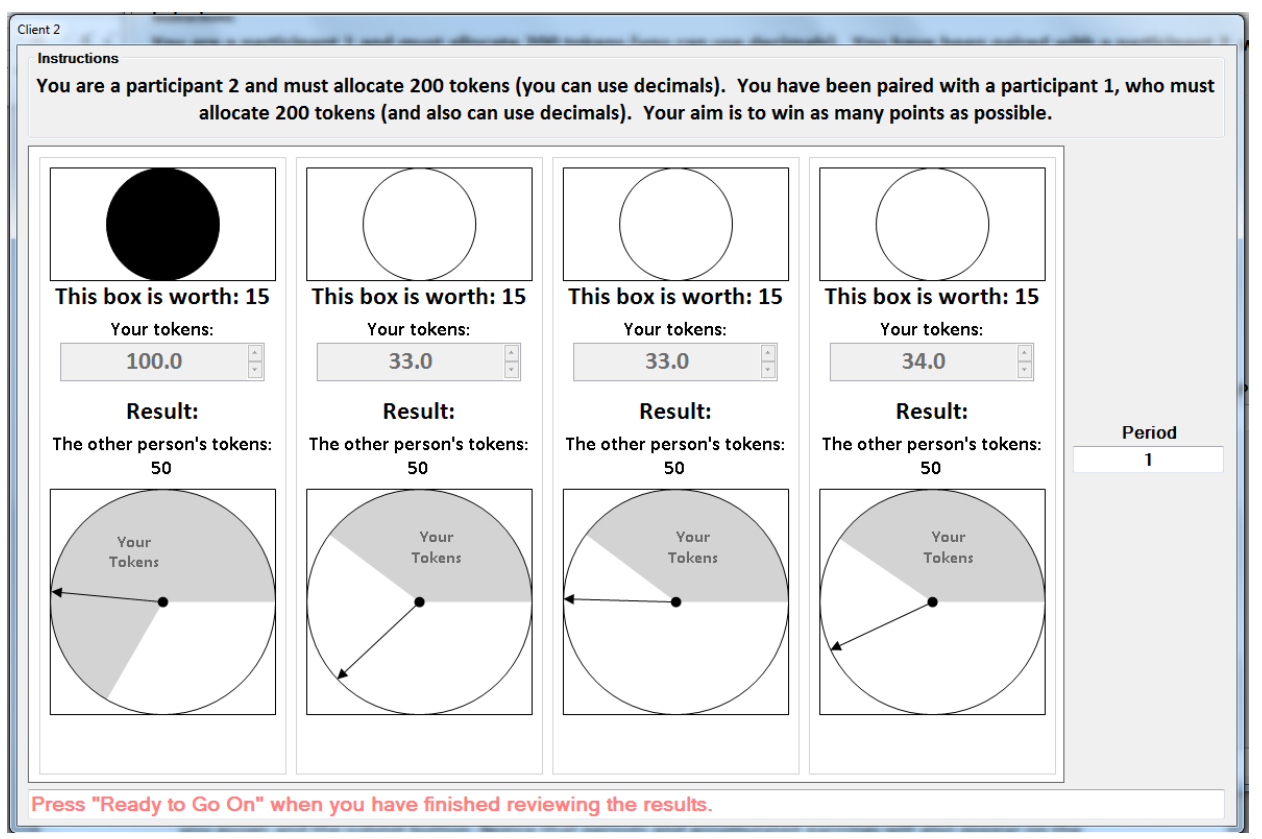

Screenshot 2: Animated spinning arrow (example of SF treatment)

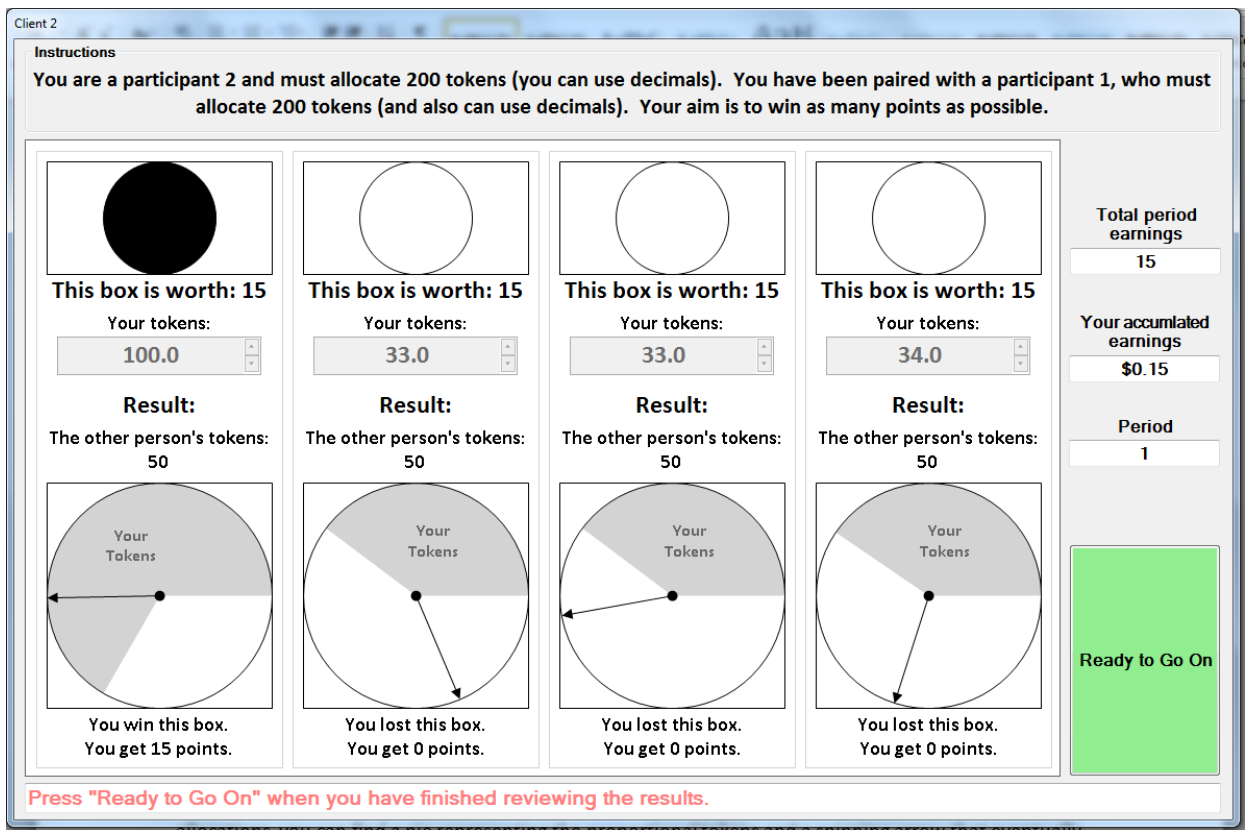

Screenshot 3: Final outcome and feedback (example of SF treatment) 


\section{Supplementary material 2 [not intended for publication]: Questionnaire}

The questionnaire below was distributed in the completely symmetric case (IS). Questionnaires for other treatments are just variations highlighted between brackets in the text.

QUESTIONNAIRE (Circle the number of the correct one)

Q1) If you are assigned as participant 1 in the first decision problem, $1=$ "you will be assigned as participant 2 in the next decision problem" 2 = "you will be assigned as participant 1 throughout the experiment [budget asymmetry treatments: (with 200 tokens)] and you will be matched to random participants 2 [budget asymmetry treatments: (with 160 tokens)]" 3 = "you will be assigned as participant 2 [budget asymmetry treatments: (with 160 tokens)] in the next decision problem and you will be matched to the same participant [budget asymmetry treatments: (with 200 tokens)]" $4=$ "you will be assigned as participant 1 throughout the experiment [budget asymmetry treatments: (with 200 tokens)] and you will be matched to the same participant 2 [budget asymmetry treatments: (with 160 tokens)]"

Q2) How do you win a particular box? $1=$ " 2 and 3 are correct" $2=$ "I win a particular box if the spinning arrow ends up on my part of the roulette" $3=$ "I might win a particular box with probability $50 \%$ when the other participant and I allocate 0 tokens" $4=$ "I will win a particular box by allocating more tokens than the other participant"

Q3) If you win 4 boxes in a certain decision problem, 1 = "you will win $\$ 4$ at the end of the experiment" $2=$ "you will win 80 points that will be converted into $\$ 3$ at the end of the experiment" $3=$ "you will win 60 points that will be converted into $\$ 3$ at the end of the experiment" $4=$ "you will win 60 points that will be converted into $\$ 3$ at the end of the experiment only if that decision problem is one of the five problems picked at the end of the experiment"

Q4) boxes are allocated randomly in a row and $1=$ " 2 and 3 are correct" $2=$ "labelled with a white circle [focal treatment: except one box which is labelled with a black circle]" $3=$ "both participants see the same row" 
Supplementary material 3 [not intended for publication]: Summary Statistics

Table SM1: Summary statistics

\begin{tabular}{|c|c|c|c|c|c|c|c|c|}
\hline \multirow[t]{2}{*}{ Statistic } & \multirow[t]{2}{*}{ Periods } & \multicolumn{7}{|c|}{ Allocation shares } \\
\hline & & IS & IF & IV & IL & AS & $\mathbf{A F}$ & AV \\
\hline & $1-10$ & & & & & & & \\
\hline $\mathrm{N}$ & & 320 & 320 & 320 & 320 & 320 & 320 & 320 \\
\hline Mean & & 0.257 & 0.282 & 0.316 & 0.194 & 0.244 & 0.262 & 0.32 \\
\hline std. dev. & & 0.126 & 0.134 & 0.134 & 0.098 & 0.102 & 0.093 & 0.182 \\
\hline $25 \%$ & & 0.2 & 0.225 & 0.25 & 0.125 & 0.2 & 0.225 & 0.25 \\
\hline 50\% (median) & & 0.25 & 0.25 & 0.3 & 0.217 & 0.25 & 0.25 & 0.334 \\
\hline \multirow[t]{2}{*}{$75 \%$} & & 0.3 & 0.35 & 0.375 & 0.25 & 0.3 & 0.3 & 0.4 \\
\hline & $11-20$ & & & & & & & \\
\hline $\mathrm{N}$ & & 320 & 320 & 320 & 320 & 320 & 320 & 320 \\
\hline Mean & & 0.256 & 0.264 & 0.317 & 0.191 & 0.252 & 0.26 & 0.339 \\
\hline std. dev. & & 0.112 & 0.113 & 0.104 & 0.097 & 0.095 & 0.096 & 0.156 \\
\hline $25 \%$ & & 0.2 & 0.2 & 0.25 & 0.125 & 0.225 & 0.218 & 0.25 \\
\hline $50 \%$ (median) & & 0.25 & 0.25 & 0.308 & 0.222 & 0.25 & 0.25 & 0.35 \\
\hline \multirow[t]{2}{*}{$75 \%$} & & 0.3 & 0.3 & 0.375 & 0.25 & 0.3 & 0.3 & 0.4 \\
\hline & All & & & & & & & \\
\hline $\mathrm{N}$ & & 640 & 640 & 640 & 640 & 640 & 640 & 640 \\
\hline Mean & & 0.257 & 0.273 & 0.317 & 0.193 & 0.248 & 0.261 & 0.33 \\
\hline std. dev. & & 0.119 & 0.124 & 0.119 & 0.097 & 0.098 & 0.094 & 0.17 \\
\hline $25 \%$ & & 0.2 & 0.2 & 0.25 & 0.125 & 0.2 & 0.22 & 0.25 \\
\hline 50\% (median) & & 0.25 & 0.25 & 0.3 & 0.22 & 0.25 & 0.25 & 0.35 \\
\hline $75 \%$ & & 0.3 & 0.325 & 0.375 & 0.25 & 0.3 & 0.3 & 0.4 \\
\hline
\end{tabular}

